\title{
Fixed-energy inverse scattering
}

\author{
A.G. Ramm \\ Mathematics Department, Kansas State University, \\ Manhattan, KS 66506-2602, USA and TU Darmstadt, Germany
}

\begin{abstract}
The author's method for solving inverse scattering problem with fixed-energy data is described. Its comparison with the method based on the D-N map is given. A new inversion procedure is formulated.
\end{abstract}

Key words: inverse scattering, fixed-energy and fixed incident direction scattering data

PACS: 03.80.+r. 03.65.Nk

1991 MSC: 35R30, 47H17, 65M30, 81U05

\section{Introduction}

The inverse scattering problem (ISP) has a long history. Fundamental results for one-dimensional (1D) inverse spectral and scattering problems were obtained by Gel'fand-Levitan, Krein and Marchenko. These results are presented in the original monographs [3], [4] and in [28], Chapter 3, where many novel results can be found, and the first presentation of Krein's theory with detailed proofs is given. The 3D ISP with fixed-energy scattering data has been open for several decades, from approximately 1943, when the question about the possibility to identify the Hamiltonian from the $S$-matrix was posed. The ISP consists of finding a potential $q=q(x)$ from the knowledge of the corresponding scattering amplitude $A\left(\alpha^{\prime}, \alpha\right)$, known at a fixed energy $k^{2}>0$ for all $\alpha^{\prime}, \alpha \in S^{2}$, where $S^{2}$ is the unit sphere in $\mathbb{R}^{3}$. One can state ISP in $\mathbb{R}^{n}$ with $n \geq 2$, but we discuss here only the case $n=3$. The results are basically the same for other $n$. Due to the lack of space we discuss the

Email address: ramm@math.ksu.edu (A.G. Ramm).

URL: http://www.math.ksu.edu/ ramm (A.G. Ramm). 
inverse potential scattering and refer to [28], [29] for inverse obstacle scattering. Let us define the scattering amplitude. Assume $q \in Q:=Q_{a} \cap L^{\infty}\left(\mathbb{R}^{3}\right)$, where $Q_{a}:=\left\{q: q(x)=\overline{q(x)}, \quad q(x) \in L^{2}\left(B_{a}\right), \quad q(x)=0\right.$ if $\left.|x| \geq a\right\}$, $B_{a}:=\{x:|x| \leq a\}, a>0$, and the overbar stands for complex conjugate. The scattering solution is the unique solution of the scattering problem:

$$
\left[\nabla^{2}+k^{2}-q(x)\right] u=0 \text { in } \mathbb{R}^{3}, \quad k=\text { const }>0, \quad u=u_{0}+v
$$

where $u_{0}:=e^{i k \alpha \cdot x}$ is the incident plane wave, $\alpha \in S^{2}$ is given, and $v$ satisfies the radiation condition: $v_{r}-i k v=o\left(r^{-1}\right)$ as $r:=|x| \rightarrow \infty$. If $q \in Q$, this implies the following asymptotic relation:

$$
v=A\left(\alpha^{\prime}, \alpha\right) \frac{e^{i k r}}{r}+o\left(r^{-1}\right), \quad r:=|x| \rightarrow \infty, \quad \alpha^{\prime}:=\frac{x}{r} .
$$

The coefficient $A\left(\alpha^{\prime}, \alpha\right):=A_{q}\left(\alpha^{\prime}, \alpha\right)$ is called the scattering amplitude. We drop its $k$-dependence of $A\left(\alpha^{\prime}, \alpha\right):=A\left(\alpha^{\prime}, \alpha, k\right)$ since $k>0$ is fixed. The ISP consists of finding $q$ from the corresponding scattering amplitude $A\left(\alpha^{\prime}, \alpha\right)$, known for all $\alpha^{\prime}, \alpha \in S^{2}$. The basic questions are:

a) Does this knowledge determine $q$ uniquely?

b) If yes, how does one calculate $q$ given $A\left(\alpha^{\prime}, \alpha\right)$ for all $\left(\alpha^{\prime}, \alpha \in S^{2}\right.$ ?

c) If $A_{\delta}\left(\alpha^{\prime}, \alpha\right)$, the "noisy data", are given, such that

$$
\sup _{\alpha^{\prime}, \alpha \in S^{2}}\left|A_{\delta}\left(\alpha^{\prime}, \alpha\right)-A\left(\alpha^{\prime}, \alpha\right)\right|<\delta
$$

how does one calculate a stable approximation to $q$, i.e., a $q_{\delta}$, such that

$$
\left\|q-q_{\delta}\right\|<\eta(\delta)
$$

where $\eta(\delta) \rightarrow 0$ as $\delta \rightarrow 0$ and $\|\cdot\|$ is some norm? How can one estimate the rate at which $\eta(\delta)$ tends to 0 ?

These three questions are: uniqueness, inversion methods for exact and noisy data, and stability of the inversion methods.

In 1987 the author proved a uniqueness theorem for 3D ISP with fixed-energy data corresponding to $q \in Q_{a}$ and gave an inversion method for exact data [11],[13]. In [17], [18] an inversion method for noisy data was developed and its error estimate was obtained for $q \in Q$. The key notion the author has introduced for the study of uniqueness of the solution to ISP and to many other inverse problems was the notion of Property $\mathrm{C}$ for a pair of differential operators, [10], [11], [12], [14], [16], [15], [23], [19] . A summary of these results was presented in [23], [25], [28]. In [6], [1], [7], [8], a method, Newton-Sabatier (NS) method, for finding a spherically-symmetric potential from the knowledge 
of the corresponding fixed-energy phase shifts was proposed. In [26] a detailed analysis of this method was given and it was proved that the NS method is fundamentally wrong in the sense that its foundations are wrong, see also [20], [24]. In [2] a uniqueness result is claimed for a a version of NS method. In [27] a counterexample to this result is given, see also [22]. An additional discussion of the NS method can be found in [20]. In Section 2 Ramm's inversion method is described. In Section 3 an inversion method which uses the Dirichlet-to Neumann (DN) map is described and compared with the author's method. The inversion method, based on the usage of DN map, was proposed in [9], see also [5], [12], [21], and [28].

\section{Ramm's inversion method for exact data}

The results in this Section are taken from [28], [18] and [25]. Let $q \in Q$ and $A\left(\alpha^{\prime}, \alpha\right)$ be the scattering amplitude at a fixed energy $k^{2}>0$. In what follows, we take $k=1$ without loss of generality. One has:

$$
A\left(\alpha^{\prime}, \alpha\right)=\sum_{\ell=0}^{\infty} A_{\ell}(\alpha) Y_{\ell}\left(\alpha^{\prime}\right), \quad A_{\ell}(\alpha):=\int_{S^{2}} A\left(\alpha^{\prime}, \alpha\right) \overline{Y_{\ell}\left(\alpha^{\prime}\right)} d \alpha^{\prime},
$$

where $S^{2}$ is the unit sphere in $\mathbb{R}^{3}, Y_{\ell}\left(\alpha^{\prime}\right)=Y_{\ell, m}\left(\alpha^{\prime}\right),-\ell \leq m \leq \ell$, are the normalized spherical harmonics, summation over $m$ is understood in (2.1) and in (2.8) below. Define the following algebraic variety in $\mathbb{C}^{3}$ :

$$
M:=\left\{\theta: \theta \in \mathbb{C}^{3}, \theta \cdot \theta=1\right\}, \quad \theta \cdot w:=\sum_{j=1}^{3} \theta_{j} w_{j} .
$$

This variety is non-compact, intersects $\mathbb{R}^{3}$ over $S^{2}$, and, given any $\xi \in \mathbb{R}^{3}$, there exist infinitely many $\theta, \theta^{\prime} \in M$ such that

$$
\theta^{\prime}-\theta=\xi, \quad|\theta| \rightarrow \infty, \quad \theta, \theta^{\prime} \in M .
$$

In particular, if one chooses the coordinate system in which $\xi=t e_{3}, t>0, e_{3}$ is the unit vector along the $x_{3}$-axis, then the vectors

$$
\theta^{\prime}=\frac{t}{2} e_{3}+\zeta_{2} e_{2}+\zeta_{1} e_{1}, \quad \theta=-\frac{t}{2} e_{3}+\zeta_{2} e_{2}+\zeta_{1} e_{1}, \quad \zeta_{1}^{2}+\zeta_{2}^{2}=1-\frac{t^{2}}{4},
$$

satisfy (2.3) for any complex numbers $\zeta_{1}$ and $\zeta_{2}$ satisfying the last equation (2.4) and such that $\left|\zeta_{1}\right|^{2}+\left|\zeta_{2}\right|^{2} \rightarrow \infty$. There are infinitely many such $\zeta_{1}, \zeta_{2} \in \mathbb{C}$. Consider a subset $M^{\prime} \subset M$ consisting of the vectors $\theta=(\sin \vartheta \cos \varphi, \sin \vartheta \sin \varphi$, $\cos \vartheta$ ), where $\vartheta$ and $\varphi$ run through the whole complex plane. Clearly $\theta \in M$, but $M^{\prime}$ is a proper subset of $M$. Indeed, any $\theta \in M$ with $\theta_{3} \neq \pm 1$ is an element of $M^{\prime}$. If $\theta_{3}= \pm 1$, then $\cos \vartheta= \pm 1$, so $\sin \vartheta=0$ and one gets 
$\theta=(0,0, \pm 1) \in M^{\prime}$. However, there are vectors $\theta=\left(\theta_{1}, \theta_{2}, 1\right) \in M$ which do not belong to $M^{\prime}$. Such vectors one obtains choosing $\theta_{1}, \theta_{2} \in \mathbb{C}$ so that $\theta_{1}^{2}+\theta_{2}^{2}=0$. There are infinitely many such vectors. The same is true for vectors $\left(\theta_{1}, \theta_{2},-1\right)$. In (2.3) one can replace $M$ by $M^{\prime}$.

Let us state two estimates proved in [28] and in [11]:

$$
\max _{\alpha \in S^{2}}\left|A_{\ell}(\alpha)\right| \leq c\left(\frac{a}{\ell}\right)^{\frac{1}{2}}\left(\frac{a e}{2 \ell}\right)^{\ell+1}
$$

and

$$
\left|Y_{\ell}(\theta)\right| \leq \frac{1}{\sqrt{4 \pi}} \frac{e^{r|I m \theta|}}{\left|j_{\ell}(r)\right|}, \quad \forall r>0, \quad \theta \in M^{\prime}
$$

where

$$
j_{\ell}(r):=\left(\frac{\pi}{2 r}\right)^{\frac{1}{2}} J_{\ell+\frac{1}{2}}(r)=\frac{1}{2 \sqrt{2}} \frac{1}{\ell}\left(\frac{e r}{2 \ell}\right)^{\ell}[1+o(1)] \text { as } \ell \rightarrow \infty,
$$

and $J_{\ell}(r)$ is the Bessel function regular at $r=0$. Note that $Y_{\ell}(\theta)$, defined above, admits a natural analytic continuation from $S^{2}$ to $M$ by taking $\vartheta$ and $\varphi$ to be arbitrary complex numbers. The resulting $\theta^{\prime} \in M^{\prime} \subset M$.

The series (2.1) converges absolutely and uniformly on the sets $S^{2} \times M_{c}$, where $M_{c}$ is any compact subset of $M$.

Fix any numbers $a_{1}$ and $b$, such that $a<a_{1}<b$. Let $\|\cdot\|$ denote the $L^{2}\left(a_{1} \leq\right.$ $|x| \leq b)$-norm. If $|x| \geq a$, then the scattering solution is given analytically:

$$
u(x, \alpha)=e^{i \alpha \cdot x}+\sum_{\ell=0}^{\infty} A_{\ell}(\alpha) Y_{\ell}\left(\alpha^{\prime}\right) h_{\ell}(r), \quad r:=|x|>a, \quad \alpha^{\prime}:=\frac{x}{r}, \quad k=1,
$$

where $A_{\ell}(\alpha)$ and $Y_{\ell}\left(\alpha^{\prime}\right)$ are defined above,

$$
h_{\ell}(r):=e^{i \frac{\pi}{2}(\ell+1)} \sqrt{\frac{\pi}{2 r}} H_{\ell+\frac{1}{2}}^{(1)}(r),
$$

$H_{\ell}^{(1)}(r)$ is the Hankel function, and the normalizing factor is chosen so that $h_{\ell}(r)=\frac{e^{i r}}{r}[1+o(1)]$ as $r \rightarrow \infty$. Define

$$
\rho(x):=\rho(x ; \nu):=e^{-i \theta \cdot x} \int_{S^{2}} u(x, \alpha) \nu(\alpha, \theta) d \alpha-1, \quad \nu \in L^{2}\left(S^{2}\right) .
$$

Consider the minimization problem

$$
\|\rho\|_{L^{2}\left(B_{b} \backslash B_{a}\right)}:=\|\rho\|=\inf :=d(\theta),
$$

where the infimum is taken over all $\nu \in L^{2}\left(S^{2}\right)$, and (2.3) holds. 
It is proved in [18], [28] that

$$
d(\theta) \leq c|\theta|^{-1} \text { if } \theta \in M, \quad|\theta| \gg 1 .
$$

The symbol $|\theta| \gg 1$ means that $|\theta|$ is sufficiently large. The constant $c>0$ in (2.11) depends on the norm $\|q\|_{L^{2}\left(B_{a}\right)}$ but not on the potential $q(x)$ itself.

An algorithm for computing a function $\nu(\alpha, \theta)$, which can be used for inversion of the exact fixed-energy three-dimensional scattering data, is as follows:

a) Find an approximate solution to (2.10) in the sense

$$
\|\rho(x, \nu)\|<2 d(\theta)
$$

where in place of the factor 2 in (2.12) one could put any fixed constant greater than 1 .

b) Any such $\nu(\alpha, \theta)$ generates an estimate $\widehat{q}$ of $\widetilde{q}(\xi)$ with the error $O\left(\frac{1}{|\theta|}\right)$, $|\theta| \rightarrow \infty$. This estimate is calculated by the formula

$$
\widehat{q}:=-4 \pi \int_{S^{2}} A\left(\theta^{\prime}, \alpha\right) \nu(\alpha, \theta) d \alpha
$$

where $\nu(\alpha, \theta) \in L^{2}\left(S^{2}\right)$ is any function satisfying (2.12).

Our basic result is:

Theorem 2.1 Let $q \in Q$ and (2.3) and (2.12) hold. Then

$$
\sup _{\xi \in \mathbb{R}^{3}}|\widehat{q}-\widetilde{q}(\xi)| \leq \frac{c}{|\theta|}, \quad|\theta| \rightarrow \infty
$$

The constant $c>0$ in (2.14) depends on the norm of $q$, but not on an individual potential $q$.

In [18], [25], and in [28], an inversion algorithm is formulated for noisy data, and the error estimate for this algorithm is obtained. Let us describe these results.

Assume that the scattering data are given with some error: a function $A_{\delta}\left(\alpha^{\prime}, \alpha\right)$ is given such that

$$
\sup _{\alpha^{\prime}, \alpha \in S^{2}}\left|A\left(\alpha^{\prime}, \alpha\right)-A_{\delta}\left(\alpha^{\prime}, \alpha\right)\right| \leq \delta
$$

We emphasize that $A_{\delta}\left(\alpha^{\prime}, \alpha\right)$ is not necessarily a scattering amplitude corresponding to some potential, it is an arbitrary function in $L^{\infty}\left(S^{2} \times S^{2}\right)$ satisfying (2.15). We assume that the unknown function $A\left(\alpha^{\prime}, \alpha\right)$ is the scattering amplitude corresponding to a $q \in Q$. 
Define $\widetilde{q}(\xi):=\int_{B_{a}} e^{-i \xi \cdot x} q(x) d x$.

The problem is:

Find an algorithm for calculating $\widehat{q}_{\delta}$ such that

$$
\sup _{\xi \in \mathbb{R}^{3}}\left|\widehat{q_{\delta}}-\widetilde{q}(\xi)\right| \leq \eta(\delta), \quad \eta(\delta) \rightarrow 0 \text { as } \delta \rightarrow 0,
$$

and estimate the rate at which $\eta(\delta)$ tends to zero.

Let us describe an algorithm for inversion of noisy data. Let

$$
N(\delta):=\left[\frac{|\ln \delta|}{\ln |\ln \delta|}\right]
$$

where $[x]$ is the integer nearest to $x>0$,

$$
\begin{gathered}
\widehat{A}_{\delta}\left(\theta^{\prime}, \alpha\right):=\sum_{\ell=0}^{N(\delta)} A_{\delta \ell}(\alpha) Y_{\ell}\left(\theta^{\prime}\right), \quad A_{\delta \ell}(\alpha):=\int_{S^{2}} A_{\delta}\left(\alpha^{\prime}, \alpha\right) \overline{Y_{\ell}\left(\alpha^{\prime}\right)} d \alpha^{\prime}, \\
u_{\delta}(x, \alpha):=e^{i \alpha \cdot x}+\sum_{\ell=0}^{N(\delta)} A_{\delta \ell}(\alpha) Y_{\ell}\left(\alpha^{\prime}\right) h_{\ell}(r), \\
\rho_{\delta}(x ; \nu):=e^{-i \theta \cdot x} \int_{S^{2}} u_{\delta}(x, \alpha) \nu(\alpha) d \alpha-1, \quad \theta \in M, \\
\mu(\delta):=e^{-\gamma N(\delta)}, \quad \gamma=\ln \frac{a_{1}}{a}>0, \\
a(\nu):=\|\nu\|_{L^{2}\left(S^{2}\right)}, \quad \kappa:=|\operatorname{Im} \theta| .
\end{gathered}
$$

Consider the following variational problem with constraints:

$$
\begin{gathered}
|\theta|=\sup :=\vartheta(\delta), \\
|\theta|\left[\left\|\rho_{\delta}(\nu)\right\|+a(\nu) e^{\kappa b} \mu(\delta)\right] \leq c, \quad \theta \in M,
\end{gathered}
$$

where the norm is defined above (2.8), and it is assumed that (2.3) holds, where $\xi \in \mathbb{R}^{3}$ is an arbitrary fixed vector, $c>0$ is a sufficiently large constant, and the supremum is taken over $\theta \in M$ and $\nu \in L^{2}\left(S^{2}\right)$ under the constraint (2.24). By $c$ we denote various positive constants.

Given $\xi \in \mathbb{R}^{3}$, one can always find $\theta$ and $\theta^{\prime}$ such that (2.3) holds. We prove that $\vartheta(\delta) \rightarrow \infty$. More precisely:

$$
\vartheta(\delta) \geq c \frac{|\ln \delta|}{(\ln |\ln \delta|)^{2}}, \quad \delta \rightarrow 0
$$


Let the pair $\theta(\delta)$ and $\nu_{\delta}(\alpha, \theta)$ be any approximate solution to problem (2.23)(2.24) in the sense that

Calculate

$$
|\theta(\delta)| \geq \frac{\vartheta(\delta)}{2}
$$

$$
\widehat{q}_{\delta}:=-4 \pi \int_{S^{2}} \widehat{A}_{\delta}\left(\theta^{\prime}, \alpha\right) \nu_{\delta}(\alpha, \theta) d \alpha .
$$

Theorem 2.2 If (2.3) and (2.26) hold, then

$$
\sup _{\xi \in \mathbb{R}^{3}}\left|\widehat{q}_{\delta}-\widetilde{q}(\xi)\right| \leq c \frac{(\ln |\ln \delta|)^{2}}{|\ln \delta|} \text { as } \delta \rightarrow 0,
$$

where $c>0$ is a constant depending on the norm of $q$.

In [18] estimates (2.14) and (2.28) were formulated with the supremum taken over an arbitrary large but fixed ball of radius $\xi_{0}$. Here these estimates are uniform with respect to $\xi$. The key point is: the constant $c>0$ in the estimate (2.11) does not depend on $\xi$.

Remark. In [21] and [28] an analysis of the approach to ISP, based on the recovery of the DN (Dirichle-to-Neumann) map from the fixed-energy scattering data, is given. This approach is discussed in Section 3.

The basic numerical difficulty of the approach, described in Theorems 2.1 and 2.2 , comes from solving problems (2.10) for exact data and problem (2.23)(2.24) for noisy data. Solving problem (2.10) amounts to finding a global minimizer of a quadratic form of the variables $c_{\ell}$, if one takes $\nu$ in (2.9) as a linear combination of the spherical harmonics: $\nu=\sum_{\ell=0}^{L} c_{\ell} Y_{\ell}(\alpha)$. If one uses the necessary condition for a minimizer of a quadratic form, that is, a linear algebraic system of equations, then the matrix of this system is ill-conditioned for large $L$. This causes the main difficulty in the numerical solution of (2.10). Methods for global minimization of the quadratic functionals, based on the gradient descent, may be also used for solving problem (2.10). Which of the two methods is more efficient only numerical experiments can tell.

Let us formulate a new method for calculating $\nu_{\delta}(\alpha, \theta)$. Consider the following minimization problem:

$$
F(\nu, \theta):=\left\|\rho_{\delta}(\nu)\right\|+a(\nu) e^{\kappa b} \mu(\delta)=\inf :=t(\delta),
$$

where the infimum is taken over $\nu \in L^{2}\left(S^{2}\right)$ and $\theta \in M$ subject to the constraint (2.3). Define $\tau(\delta):=\frac{(\ln |\ln \delta|)^{2}}{|\ln \delta|}$.

Theorem 2.3 Under the assumptions of Theorem 2.1 one has:

$$
t(\delta)=O(\tau(\delta)) \text { as } \delta \rightarrow 0 .
$$


Theorem 2.4 If the pair $\nu_{\delta}, \theta(\delta)$ satisfies the inequality $F\left(\nu_{\delta}, \theta(\delta)\right)<2 t(\delta)$, then (2.28) holds, where $\widehat{q}_{\delta}$ is defined in (2.27).

The key point in the proof are the following two estimates:

$$
\begin{aligned}
& \left\|\rho_{\delta}(\nu)\right\|+a(\nu) e^{\kappa b} \mu(\delta) \leq c\left[\|\rho(\nu)\|+a(\nu) e^{\kappa b} \mu(\delta)\right], \\
& \|\rho(\nu)\|+a(\nu) e^{\kappa b} \mu(\delta) \leq c\left[\left\|\rho_{\delta}(\nu)\right\|+a(\nu) e^{\kappa b} \mu(\delta)\right],
\end{aligned}
$$

estimate (2.11), estimate $a(\nu) \leq e^{c s \ln s}$, where $s:=|\theta|$ and $\nu$ is such that inequality (2.11) holds (see inequality (2.23) in[25]), and the following result: $\min _{s>0}\left[\frac{1}{s}+e^{c s \ln s+b s} \mu(\delta)\right]=O(\tau(\delta))$ as $\delta \rightarrow 0$.

\section{Discussion of the inversion method which uses the DN map}

In [21] the following inversion method is discussed:

$$
\tilde{q}(\xi)=\lim _{|\theta| \rightarrow \infty} \int_{S_{a}} \exp \left(-i \theta^{\prime} \cdot s\right)\left(\Lambda-\Lambda_{0}\right) \psi d s,
$$

where (2.3) is assumed, $\tilde{q}(\xi):=\int_{B_{a}} e^{-i \xi \cdot x} q(x) d x, S_{a}$ is a sphere of radius $a$, centered at the origin, $\Lambda$ is the Dirichlet-to-Neumann (DN) map, $\psi$ is found from the equation:

$$
\psi(s)=\psi_{0}(s)-\int_{S_{a}} G(s-t) B \psi d t, \quad B:=\Lambda-\Lambda_{0}, \quad \psi_{0}(s):=e^{i \theta \cdot s},
$$

and $G$ is defined by the formula:

$$
G(x)=\exp (i \theta \cdot x) \frac{1}{(2 \pi)^{3}} \int_{\mathbb{R}^{3}} \frac{\exp (i \xi \cdot x) d \xi}{\xi^{2}+2 \xi \cdot \theta} .
$$

The DN map is constructed from the fixed-energy scattering data $A\left(\alpha^{\prime}, \alpha\right)$ by the method of [21] (see also [28]).

Namely, given $A\left(\alpha^{\prime}, \alpha\right)$ for all $\alpha^{\prime}, \alpha \in S^{2}$, one finds $\Lambda$ using the following method.

Let $f \in H^{3 / 2}(S)$ be given and $f_{\ell}$ are its Fourier coefficients in the basis of the spherical harmonics,

$$
w=\sum_{l=0}^{\infty} f_{l} Y_{l}\left(x^{0}\right) \frac{h_{l}(r)}{h_{l}(a)}, \quad r \geq a, \quad x^{0}:=\frac{x}{r}, \quad r:=|x| .
$$


Let

$$
w=\int_{S_{a}} g(x, s) \sigma(s) d s,
$$

where $\sigma$ is some function, which we find below, and $g$ is Green's function (resolvent kernel) of the Schrödinger operator, satisfying the radiation condition at infinity. The DN map is the map $\Lambda: f \rightarrow w_{N}^{+}$, where $N$ is the outer normal to $S_{a}$, and $w_{N}^{+}$is the normal derivative on $S_{a}$ from the interior of $B_{a}$ along the radius-vector. By the jump formula for the normal derivative of a single-layer potential one has:

$$
w_{N}^{+}=w_{N}^{-}+\sigma .
$$

We require $w=f$ on $S_{a}$. Then $w$ is given by formula (3.4) in the exterior of $S_{a}$, and

$$
w_{N}^{-}=\sum_{l=0}^{\infty} f_{l} Y_{l}\left(x^{0}\right) \frac{h_{l}^{\prime}(a)}{h_{l}(a)} .
$$

By formulas (3.6) and (3.7), finding $\Lambda$ is equivalent to finding $\sigma$. By (3.5), asymptotics of $w$ is (cf [28], p.257):

$$
w=\frac{e^{i r}}{r} \frac{u\left(y,-x^{0}\right)}{4 \pi}+o\left(\frac{1}{r}\right), \quad r:=|x| \rightarrow \infty, \quad \frac{x}{|x|}:=x^{0},
$$

where $u$ is the scattering solution,

$$
u\left(y,-x^{0}\right)=e^{-i x^{0} \cdot y}+\sum_{\ell=0}^{\infty} A_{\ell}\left(-x^{0}\right) Y_{\ell}\left(y^{0}\right) h_{\ell}(|y|) .
$$

From (3.4), (3.8) and (3.9) one gets an equation for finding $\sigma$ ([21] eq. (23)), see also [28], p.295):

$$
\frac{f_{l}}{h_{l}(a)}=\frac{1}{4 \pi} \int_{S_{a}} d s \sigma(s)\left(u(s,-\beta), Y_{l}(\beta)\right)_{L^{2}\left(S^{2}\right)} .
$$

This equation can be written as a linear system:

$$
\frac{4 \pi f_{l}}{h_{l}(a)}=a^{2}(-1)^{l} \sum_{l^{\prime}=0}^{\infty} \sigma_{l^{\prime}}\left[4 \pi i^{l} j_{l}(a) \delta_{l l^{\prime}}+A_{l^{\prime} l} h_{l^{\prime}}(a)\right]
$$

for the Fourier coefficients $\sigma_{\ell}$ of the function $\sigma(s)$. The coefficients

$$
A_{l^{\prime} l}:=\left(\left(A\left(\alpha^{\prime}, \alpha\right), Y_{\ell}\left(\alpha^{\prime}\right)\right)_{L^{2}\left(S^{2}\right)}, Y_{\ell}(\alpha)\right)_{L^{2}\left(S^{2}\right)}
$$

are the Fourier coefficients of the scattering amplitude. Problems (3.10) and (3.11) are very ill-posed (see [21] for details).

This approach faces several serious difficulties:

1) The construction of the DN map from the scattering data is a very ill-posed problem, 
2) The construction of the potential from the DN map is a very difficult problem numerically, because one has to solve a Fredholm-type integral equation (equation (3.2)) whose kernel contains $G$, defined in (3.3). This $G$ is a tempered distribution, and it is not clear how to compute it accurately,

3) One has to calculate a limit of an integral whose integrand grows exponentially to infinity if a factor in the integrand is not known exactly. The solution of equation (3.2) is one of the factors in the integrand. It cannot be known exactly in practice because it cannot be calculated with arbitrary accuracy even if the scattering data are known exactly. Therefore the limit in formula (3.1) cannot be calculated accurately.

No error estimates are obtained for this approach.

In contrast, in Ramm's method, there is no need to compute $G$, to solve equation (3.2), to calculate the DN map from the scattering data, and to compute the limit (3.1). The basic difficulty in Ramm's inversion method for exact data is to minimize a quadratic form (2.10), and for noisy data to solve optimization problem (2.29) or (2.23)-(2.24). The error estimates are obtained for the Ramm's method.

\section{References}

[1] Chadan K., Sabatier P., Inverse Problems in Quantum Scattering Theory, (Springer, New York, 1989).

[2] Cox, J., Thompson, K., Note on the uniqueness of the solution of an equation of interest in the inverse scattering problem, 1970, J. Math. Phys., 11, N3, 815-817.

[3] Levitan, B., Inverse Sturm-Liouville problems, (VNU Press, Utrecht, 1987).

[4] Marchenko, V., Sturm-Liouville operators and applications, (Birkhäuser, Boston,1986).

[5] Nachman, A., Reconstruction from boundary measurements, 1988, Ann. Math., 128, N3, 531-576.

[6] Newton R., Scattering theory of waves and particles (Springer, New York, 1982).

[7] Newton R., Construction of potentials from phase shifts at fixed energy, 1962, J. Math. Phys., 3, N1, 75-82.

[8] Newton R., Connection between complex angular momenta and the inverse scattering problem, 1967, J.Math. Phys., 8, N8, 1566-1570.

[9] Novikov, R.G., Multidimensional inverse spectral problem for the Schrödinger equation, 1988, Func. Anal. Appl., 22, N4, 263-272. 
[10] Ramm, A.G., On completeness of the products of harmonic functions, 1986, Proc. A.M.S., 99, 253-256.

[11] Ramm, A.G., Completeness of the products of solutions to PDE and uniqueness theorems in inverse scattering, 1987, Inverse problems, 3, L77-L82.

[12] Ramm, A.G., Multidimensional inverse problems and completeness of the products of solutions to PDE, 1988, J. Math. Anal. Appl. 134, 1, 211-253; 139, 302 (1989).

[13] Ramm, A.G., Recovery of the potential from fixed energy scattering data, 1988, Inverse Problems, 4, 877-886.

[14] Ramm, A.G., Multidimensional inverse scattering problems and completeness of the products of solutions to homogeneous PDE, 1989, Zeitschr. f. angew. Math. u. Mech., 69, N4, T13-T22.

[15] Ramm, A.G., Stability of the numerical method for solving the 3D inverse scattering problem with fixed energy data, 1990, Inverse problems 6, L7-12.

[16] Ramm, A.G., Completeness of the products of solutions of PDE and inverse problems, 1990, Inverse Probl., 6, 643-664.

[17] Ramm, A.G., Stability of the numerical method for solving 3D inverse scattering problem with fixed energy data, 1991, Jour. fuer die reine und angew. Math., 414, 1-21.

[18] Ramm, A.G., Stability estimates in inverse scattering, 1992, Acta Appl. Math., $28, \mathrm{~N} 1,1-42$.

[19] Ramm, A.G., Stability of the inversion of 3D fixed-frequency data, 1992, J. Math. Anal. Appl., 169, N2, 329-349.

[20] Ramm, A.G., Comments on the letter of P.Sabatier, ArXiv PaperId: mathph/0308025, http://arXiv.org/abs/math-ph/0308025.

[21] Ramm, A.G., Finding potential from the fixed-energy scattering data via D-N map, 1996, J. of Inverse and Ill-Posed Problems, 4, N2, 145-152.

[22] Ramm, A.G., Inverse scattering problem with part of the fixed-energy phase shifts, 1999, Comm. Math. Phys. 207, N1, 231-247.

[23] Ramm, A.G., Property C for ODE and applications to inverse problems, in the book "Operator Theory and Its Applications", Amer. Math. Soc., Fields Institute Communications vol. 25, pp.15-75, editors A.G.Ramm, P.N.Shivakumar, A.V.Strauss (Providence, RI. 2000).

[24] Ramm, A.G., Gutman, S., Piecewise-constant positive potentials with practically the same fixed-energy phase shifts, 2001, Applicable Analysis, 78, N1-2, 207-217.

[25] Ramm, A.G., Stability of the solutions to 3D inverse scattering problems, 2002, Milan Journ of Math 70, 97-161. 
[26] Ramm, A.G., Analysis of the Newton-Sabatier scheme for inverting of the fixedenergy phase shifts, 2002, Applic. Analysis, 81, N4, 965-975.

[27] Ramm, A.G., A counterexample to the uniqueness result of Cox and Thompson, 2002, Applic. Analysis, 81, N4, 833-836.

[28] Ramm, A.G., Inverse problems (Springer, New York, 2005).

[29] Ramm, A.G., Scattering by obstacles (D. Reidel, Dordrecht, 1986). 